\title{
PERÚ: PANDEMIA Y CORRUPCIÓN
}

\author{
Peru: pandemic and corruption
}

\author{
Ketty Marilú Moscoso Paucarchuco ${ }^{4}$ \\ Universidad Nacional Autónoma de Huanta, Perú.
}

\section{RESUMEN}

El presente capítulo, busca analizar cualitativamente la trascendencia del Objetivo Sostenible 16 de la Agenda 2030, referido a reducir considerablemente la corrupción y su incidencia durante el periodo de pandemia en el Perú, esta investigación de tipo básica, no experimental, descriptiva; permitió observar y medir las variables, determinando sus características de manera estadística, bajo el método

4 Investigadora RENACYT María Rostworowski - Nivel III, Docente Ordinario de la Universidad Nacional Autónoma de Huanta, Doctorando en Ciencias Contables y Empresariales, Maestra en Gestión Pública, Contador Público Colegiado, Bachiller en Derecho y Ciencias Políticas, egresada de la carrera de Administración y Negocios Internacionales, estudios culminados de Segunda Especialización Profesional en Tecnologías de la Información y Comunicación, con diplomados y cursos diversos, miembro del Instituto de Investigación de la Universidad Nacional Autónoma de Huanta, Investigadora Principal y Co-Investigadora en Proyectos de Investigación con Fondos Concursables - FOCAM y proyectos de investigación autofinanciados, con producción científica publicada, revisora por pares de artículos científicos en revistas indexadas, formuladora y evaluadora de proyectos de investigación, integrante del Consejo Directivo de la Revista "Empresa para todos" de la Universidad Nacional del Centro del Perú, columnista semanal en el "Periódico Digital unahALDIA", parte del equipo (I+D) de creación de sistemas inéditos que contribuyen a gestión universitaria, patentados en INDECOPI. Con más de 15 años de labor profesional, amplia experiencia en el sector público y privado, asumiendo cargos gerenciales y directivos a nivel Local, Regional y Nacional. Registro Orcid: https://orcid.org/0000-0003-2097-8658, Scopus Author ID: 57219243602, correspondencia a kmoscoso@ unah.edu.pe 
inductivo-deductivo de carácter fenomenológico y enfoque hermenéutico.

La información obtenida de las diversas páginas de transparencia del gobierno nacional, regional y local, así como información investigativa, académica y periodística han permitido concluir que la corrupción es una problemática social cuya estrategia de eliminación debe ser transversal dentro de los Objetivos del Desarrollo Sostenible (ODS) planeados al 2030, que su impacto ha dañado social y económicamente a nuestro país, reflejándose en debilidades para confrontar contingencias de fuerza mayor como la pandemia.

La lucha contra la corrupción implica un trabajo integral, controlar la ejecución de S/.22 864873 138.00, resulta una tarea titánica, que no se debe encomendar a un solo ente rector, prima mucho el principio de trasparencia y buena fe; mejorar y hacer más ágil, el marco legal en el aspecto administrativo, civil y penal; elaborar estrategias como los ODS, resultan importantes, pero sin una premisa de eliminación de la corrupción que se aplique a los 17 objetivos, veremos truncadas las expectativas; este mal endeble trae consigo pobreza, hambre, carencia en educación y salud, ausencia de agua limpia y saneamiento, contaminación y un sinfín que amenazas para el logro de una nación sostenible, que mantenga un equilibrio entre lo económico, social y ambiental.

\section{INTRODUCCIÓN}

La pandemia ha aflorado las más grandes debilidades que tiene nuestro país, al encontrarnos desarmados para enfrentar a un enemigo invisible ante nuestros ojos, pero poderoso; sin las condiciones básicas en salud, educación, empleo, tecnología, entre otros; el resultado ha sido atroz; la pérdida de vidas y las secuelas de enfermedad han dejado una huella imborrable, un desplome económico a causa de las interrupciones de las cadenas de pagos y producción, fuga de capitales y devaluación de monedas han ubicado 
a nuestra economía en el record de disminución del producto bruto interno después de décadas; del 2019 al 2020 en el primer trimestre se disminuyó $3,5 \%$; en el segundo trimestre $29,8 \%$; en el tercer trimestre $9,4 \%$, acumulando a la fecha una caída abismal de 14,5\% (Carhuavilca Bonett, Dante; Sánchez Aguilar, Aníbal; Robles Luis, José; Meza Meza, 2020).

Bajo este panorama ¿dónde quedo el desarrollo sostenible de Perú? ¿cuál fue el error que se cometió para que nuestros bienes y servicios estatales no hayan cumplido eficientemente su función? ¿la corrupción fue el problema? son interrogantes que se responden a lo largo del presente escrito.

Dentro de las metas de los ODS de la Agenda 2030, se tiene casi de manera residual dentro del Objetivo 16, el acápite referido a reducir considerablemente la corrupción y el soborno en todas sus formas, mencionando que resulta loable que para promover sociedades pacíficas e inclusivas, se facilite el acceso a la justicia para todos, generando instituciones eficaces e inclusivas y que además rindan cuentas (Naciones Unidas/CEPAL, 2019). Filtrar esta agenda implica la falta de una apelación directa a la lucha contra la corrupción, sobre todo cuando ya se ha demostrado empíricamente la correlación (Tapia Gutiérrez, 2016) con distintas variables dentro de esta misma agenda, como: pobreza, hambre, salud, educación, trabajo, igualdad, producción, consumo, protección del medio ambiente, etc. Es importante considerar que el trabajo transparente y honesto de gestiones gubernamentales solucionaría la mayoría de problemas que impiden que nuestro país sea sostenible.

Es lamentable pero realista afirmar que, la corrupción es percibida como una anomalía cotidiana, un quebrantamiento del funcionamiento normal de las instituciones, incluso como hechos propios de la idiosincrasia nacional (Ramón R, 2014), son estas las razones por las cuales se aborda esta problemática, la corrupción genera impacto negativo, desvirtuando la democracia y la 
gobernabilidad; sin considerar los derechos humanos, las garantías constitucionales, la ética; estas prácticas nocivas, deben ser jurídicamente controladas en vía administrativa, civil y penal; la pandemia ha ocasionado que la ciudadanía ya no espere en actitud pasiva. Es inexorable recuperar la confianza del ciudadano, para ello se requieren estrategias como los ODS que debieran incorporar de manera transversal la eliminación de la corrupción como pilar fundamental de la sostenibilidad de las naciones.

El presente estudio de carácter básico, cualitativo, no experimental, descriptivo, inductivo, deductivo, fenomenológico y hermenéutico ha permito el análisis de la pandemia y la corrupción en términos asociado a los ODS propuestos en la Agenda 2030, para lo cual se ha conceptualizado la corrupción; se ha debatido sobre los métodos que se aplican para eliminarla, se ha analizado la asignación del presupuesto nacional por tema de pandemia, así como los antecedentes e índices de corrupción y las diversas situaciones denunciadas de manera pública durante el periodo 2020.

\section{CORRUPCIÓN}

La administración pública es un bien jurídico, cuya protección está prescrita en la normatividad. Se considera injusticia cuando un funcionario público quebranta la finalidad del uso de un bien o prestación de un servicio del Estado, situando este, en favor personal; azotando vertiginosamente la confianza de la ciudadanía, que espera una sanción punible, que resulta blanda y para su sentencia, tarda mucho más que un delito común. Los hechos de corrupción en las últimas décadas se han enquistado en las altas esferas del poder, para contrarrestar se ha generado un sistema anticorrupción, débil y endeble frente a las estrategias de criminalidad de los conocidos cuellos blancos.

Dar una definición a este tipo de delito es complejo, la ley tampoco es clara, pues no tipifica las conductas que conforman la idea de corrupción, siendo difícil calcular todos los malos usos del poder 
con el fin de alcanzar una ventaja ilegal. La corrupción es aquel que la concibe como abuso de poder público para la obtención de un beneficio particular. En otras palabras, el "fenómeno por medio del cual un funcionario público es impulsado para favorecer intereses particulares a cambio de una recompensa" (Rodríguez Collao, 2004).

Etimológicamente, corrupción viene del latín corruptio que significa, entre otras cosas, corrupción, alteración, seducción. Este sustantivo viene del verbo corrumpo cuyo significado es múltiple: corromper, echar a perder, alterar, falsificar, manchar, marchitar, descomponer, teñir, perder, arruinar, destruir, roer, disipar, pervertir, depravar, seducir, cohechar, sobornar, alterar las ideas de alguien, deshonrar. Este verbo viene de cum y rumpo. Rumpo es romper, destrozar, desgarrar, deshacer, quebrar, quebrantar, partir, violentar, ahogar, hender, separar, abrir, reventar, penetrar, violar, hacer brotar, interrumpir, prorrumpir, dejar escapar. Cum es con y siempre significa variedad de relaciones. Si unimos todas estas acepciones, el resultado es muy significativo: degeneración de las costumbres ciudadanas (Soto Posada, 2018).

El hecho de corrupción por su propia complejidad a nivel económico y jurídico, no resulta fácil de perseguir por los órganos judiciales, es por ello que su tratativa se da a nivel de salas especializadas como la Fiscalía Anticorrupción de Funcionarios, quienes tienen la competencia para infiltrarse en estas redes que simulan a un crimen organizado. La corrupción impide, es un obstáculo para el logro de los ODS.

Si existe corrupción no habrá eliminación de la pobreza, se tendrá hambre, empeorará la salud y el bienestar, no habrá una educación de calidad, seguirán las diferencias entre varones y mujeres, no se tendrá agua limpia ni saneamiento, la contaminación invadirá, el crecimiento económico y el trabajo decente estarán lejanos, la industria con su innovación e infraestructura no avanzará, no habrán 
instituciones sólidas, mucho menos paz y justicia; en resumen con corrupción se está lejano a lograr la sostenibilidad.

\section{MÉTODOS PARA LA ELIMINACIÓN DE LA CORRUPCIÓN}

La corrupción representa una amenaza significativa para los países del mundo entero; debilita las instituciones democráticas, contribuye a la inestabilidad gubernamental y erosiona la confianza (UNODC, 2018). La corrupción generalizada es síntoma de una sociedad severamente enferma con una mayoría de recursos e innovación asignada al área de redistribución, es entorpecer del futuro de la nación. Y es la nación la que debe tomar algunas decisiones difíciles al respecto (Begovic, 2005).

Para luchar contra la corrupción es vital adoptar un enfoque integral, que enmarque transparencia, rendición de cuentas y participación colectiva; cuanto más control haya de esa variable, mejor entorno de inversión y más crecimiento económico habrá (Rodriguez Soria, Beatriz; Jacotte Simancas, Coro; Moreno Fernández, José Ramón; Álvarez Domínguez, 2016).

Desde el gobierno central se debe impulsar reformas legislativas sólidas que adopten medidas sancionables claras; la prevención es significativa; por ello es sustancial considerar un personal idóneo para la toma de decisiones, con códigos de buenas prácticas gubernamentales; fortalecimiento de la académica política, con un plan de formación ciudadana que busque transparencia y democracia, evaluación detallada el financiamiento a los partidos políticos (es aquí donde nace la corrupción), prohibición de aportes económicos de personas jurídicas a campañas electorales, publicación de los aportes en las campañas, fortalecimiento la Oficina de Procesos Electorales; son propuestas considerables. El objetivo de estas medidas es luchar contra la corrupción, profundizar la democracia y la transparencia, así como recuperar la confianza de la ciudadanía en la actividad política (Gobierno de Chile, 2017). 
Mejorar los estándares de transparencia, desarrollar un sistema preventivo anti corrupción, usar de forma obligatoria de portales para la información a la ciudadanía, respeto a la ejecución del presupuesto institucional, en el área de logística para conocer a los proveedores y su precio de oferta, en el área de personal, para tener acceso a empleos, con procesos meritocráticos y transparentes, en el área de control, para evaluar la rendición de cuentas y su sustentación respecto al cumplimiento de la finalidad pública, etc.

Debido a la pandemia de COVID-19, los países han intensificado el gasto, en especial el gasto contingente para responder a las necesidades urgentes relacionadas a salvar vidas y generar medios de subsistencia (Khasiani et al., 2020). Para garantizar la eficacia y eficiencia de dicho gasto o inversión, es trascendental tomar consciencia de la cantidad en términos monetarios del presupuesto distribuido a nivel nacional, regional y local; dinero que se encuentra ante la vulnerabilidad de actos de corrupción.

\section{ASIGNACIÓN DEL PRESUPUESTO NACIONAL PARA COMBATIR LA PANDEMIA}

La asignación presupuestal por motivo de COVID 19, en niveles de gobierno ascendieron a un total de S/.22 864873 138,00, distribuyendo para el Gobierno Nacional un promedio del 90\%, para el Gobierno Regional un $8 \%$ y para el Gobierno Local un 2\%; alcanzando un nivel de ejecución de gasto entre los tres niveles de gobierno de un $80 \%$, según la Tabla 1. 


\section{Tabla 1.}

Asignación y ejecución presupuestal COVID-19, por niveles de Gobierno.

\begin{tabular}{crrr}
\hline Nivel de Gobierno & Presupuesto asignado & Presupuesto ejecutado & Avance \% \\
\hline Gobierno Nacional & S/20 363533484,00 & S/16 453080863,00 & $81 \%$ \\
Gobierno Regional & S/1 861003639,00 & S/1 406704142,00 & $76 \%$ \\
Gobierno Local & S/640 336 015,00 & S/486 550 447,00 & $76 \%$ \\
Total & S/22 864 873 138,00 & S/18 346 335 452,00 & $\mathbf{8 0 \%}$ \\
\hline
\end{tabular}

Fuente: Adecuado de (MEF, 2020) al 06 de diciembre del 2020.

Analizando estos resultados, se puede manifestar que existe una centralización casi total del presupuesto, certificando que los recursos por motivo de COVID 19, fueron administrados por el Gobierno Nacional. Dejando de lado el proceso de descentralización, que debe llevarse de manera "progresiva y ordenada", que permita una transferencia presupuestal salomónica y equitativa, distribuyendo poderes de decisión y ejecución; este problema no permite el crecimiento en las distintas regiones, pues sus representantes extenuados esperan gota a gota el presupuesto, el gobierno central cree conocer todas las necesidades del pueblo, en su entorno citadino y alejado de la realidad provinciana. Los Gobiernos Regionales y Locales, tuvieron una participación mínima en la ejecución del presupuesto, ver Figura 1, así que para medir la calidad del gasto se tiene que poner énfasis en los entes de administración nacional principalmente, para ser específicos en los diversos ministerios del estado. 


\section{Figura 1.}

Asignación y ejecución presupuestal COVID-19, por niveles de Gobierno.

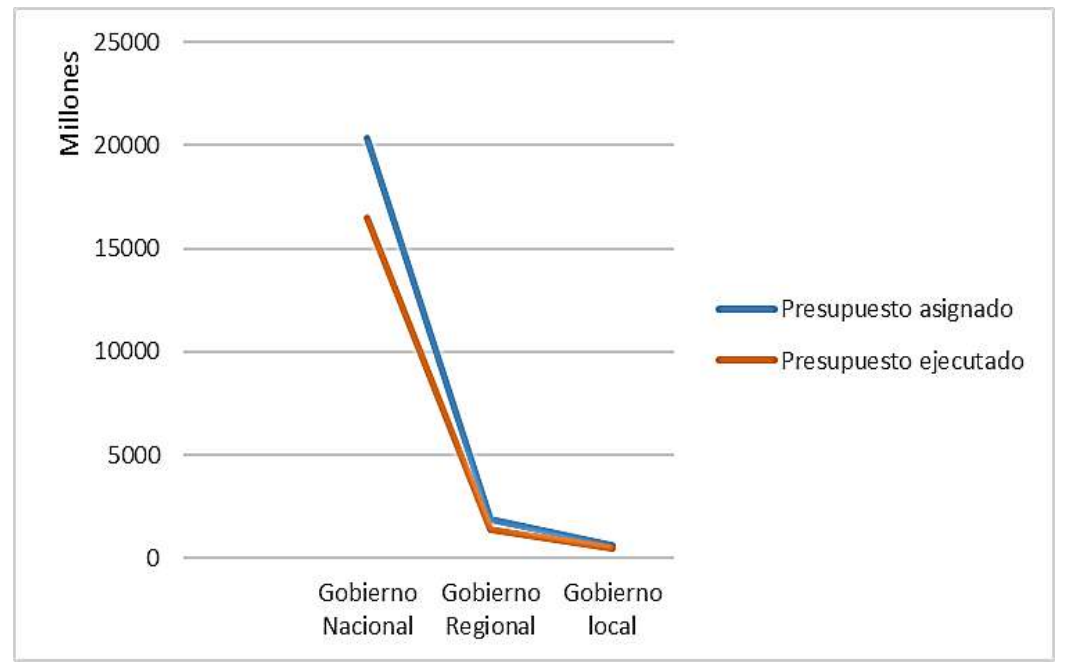

Analizar esta distribución por regiones, deja en evidencia la inequidad, Lima obtuvo el $53 \%$ del presupuesto del Gobierno Nacional, seguido de Cajamarca y Piura con 4\%; en el Gobierno Regional, La libertad y Piura obtuvieron el 7\% del presupuesto, seguido de Cusco, Junín, San Martín y Áncash con el 6\%; en el Gobierno Local, la distribución favoreció a Lima con un 27\%, seguido de la Libertad con 7\%, Puno, Piura, Cajamarca y Ancash con un 6\%.

Del resumen general de los tres niveles de gobierno, se determina que Lima cuenta con una asignación equivalente al 48\%, ¿será cierto que casi mitad del país viven el Lima? con un $4 \%$ tenemos a Cajamarca, Cusco, La Libertad y Piura; las asignaciones más pequeñas (1\% promedio), se dirigieron Madre de Dios, Moquegua, Tumbes, Pasco, Tacna, Huancavelica, Apurímac, Ucayali y Amazonas, ver Tabla y Figura 2. 
En el último censo nacional de 2017, Lima tenía una medición poblacional del 32\%, seguidos de Piura y La libertad con 6\%; y Arequipa y Cajamarca con 4\%; dentro de las Regiones menos pobladas tenemos a Apurímac, Amazonas, Huancavelica, Tacna, Pasco, Tumbes y Moquegua. Considerando este análisis se puede apreciar que Lima tiene una asignación económica superior a su índice poblacional, impidiendo un crecimiento en la gestión gubernamental descentralizada.

\section{Tabla 2.}

Asignación y ejecución presupuestal COVID-19, por niveles de Gobierno a las Regiones.

\begin{tabular}{|c|c|c|c|c|}
\hline Regiones & Gobierno nacional & Gobiernos Regional & Gobierno Local & Total \\
\hline Amazonas & $\mathrm{S} / 274690158,00$ & S/42 441514,00 & S/9 893 648,00 & $\mathrm{S} / 327025320,00$ \\
\hline Ancash & S/485 488 782,00 & S/117 172205,00 & S/39 097947,00 & S/641 758934,00 \\
\hline Apurímac & $\mathrm{S} / 245313432,00$ & $\mathrm{~S} / 46085$ 128,00 & S/11 921260,00 & $\mathrm{~S} / 303319820,00$ \\
\hline Arequipa & $\mathrm{S} / 550499704,00$ & S/82 180374,00 & 168,00 & $S / 65669$ \\
\hline Ayacucho & S/339 483244,00 & S/75 262592,00 & S/19 220 155,00 & S/433 965991,00 \\
\hline Cajamarca & 1 106,00 & S/98 128966,00 & 300,00 & S/951 605872,00 \\
\hline Callao & $S / 39$ & $S / 819$ & 0,00 & $\mathrm{~S} / 4842$ \\
\hline Cusco & S/665 041662,00 & S/106 879 858,00 & $S / 33313500,00$ & $\mathrm{~S} / 805235020,00$ \\
\hline Huancavelica & $S / 2231$ & S/61 18 & $S / 13$ & $\mathrm{~S} / 2980$ \\
\hline Huánuco & S/39691 & $S / 683$ & $S / 15$ & $\mathrm{~S} / 480769203,00$ \\
\hline Ica & 60,00 & $S / 631$ & $S / 13$ & $S / 3762$ \\
\hline Junín & $S / 60$ & $S / 1120$ & & S/7379 \\
\hline La Libertad & 91,00 & $S / 1231$ & $S / 44$ & S/871 283041,00 \\
\hline Lambayeque & 0,00 & 00 & $S / 23$ & $S / 5782$ \\
\hline Lima & $S / 1070$ &, 00 & $S / 173$ & S/109622 \\
\hline Loreto & 203,00 & S/85 6 & $S / 18551264,00$ & S/676 990052,00 \\
\hline Madre De Di & 00 &, 00 & 00 & $S / 1020$ \\
\hline Moquegua & 7,00 & S/497 &, 00 & $\mathrm{~S} / 1250$ \\
\hline Pasco & 99,00 & $S / 287$ & 00 & $S / 1741$ \\
\hline Piura & & S/131 713991,00 & $S / 36$ & S/889 544096,00 \\
\hline Puno & & 105,00 & $S / 36$ & $S / 7745$ \\
\hline San Martin & & S/112 962066,00 & & $\mathrm{~S} / 557263976,00$ \\
\hline Tacna & & S/36 988544,00 & S/12 526374,00 & S/191 100063,00 \\
\hline Tumbes & S/92 781 150,00 & $\mathrm{S} / 34150$ 111,00 & S/3 112277,00 & $\mathrm{~S} / 1300$ \\
\hline & & S/50 931 016,00 & S/8 286506,00 & $\mathrm{~S} / 304234459,00$ \\
\hline Exterior & S/31 247500,00 & $\mathrm{~S} / 0,00$ & $\mathrm{~S} / 0,00$ & S/31 247500,00 \\
\hline Total & 20363533 484,00 & $S / 1861003639,00$ & $\mathrm{~S} / 640336015,00$ & $\mathrm{~S} / 22864873138,00$ \\
\hline
\end{tabular}

Fuente: Adecuado de $(M E F, 2020)$ al 06 de diciembre del 2020. 
Dentro de la distribución presupuestal también podemos hallar un presupuesto al exterior del país, que ha sido asignado para prevención, control, diagnóstico y tratamiento de coronavirus, en las partidas de bienes y servicios, y donaciones y transferencias.

\section{Figura 2.}

Asignación y ejecución presupuestal COVID-19, por niveles de Gobierno a las Regiones.

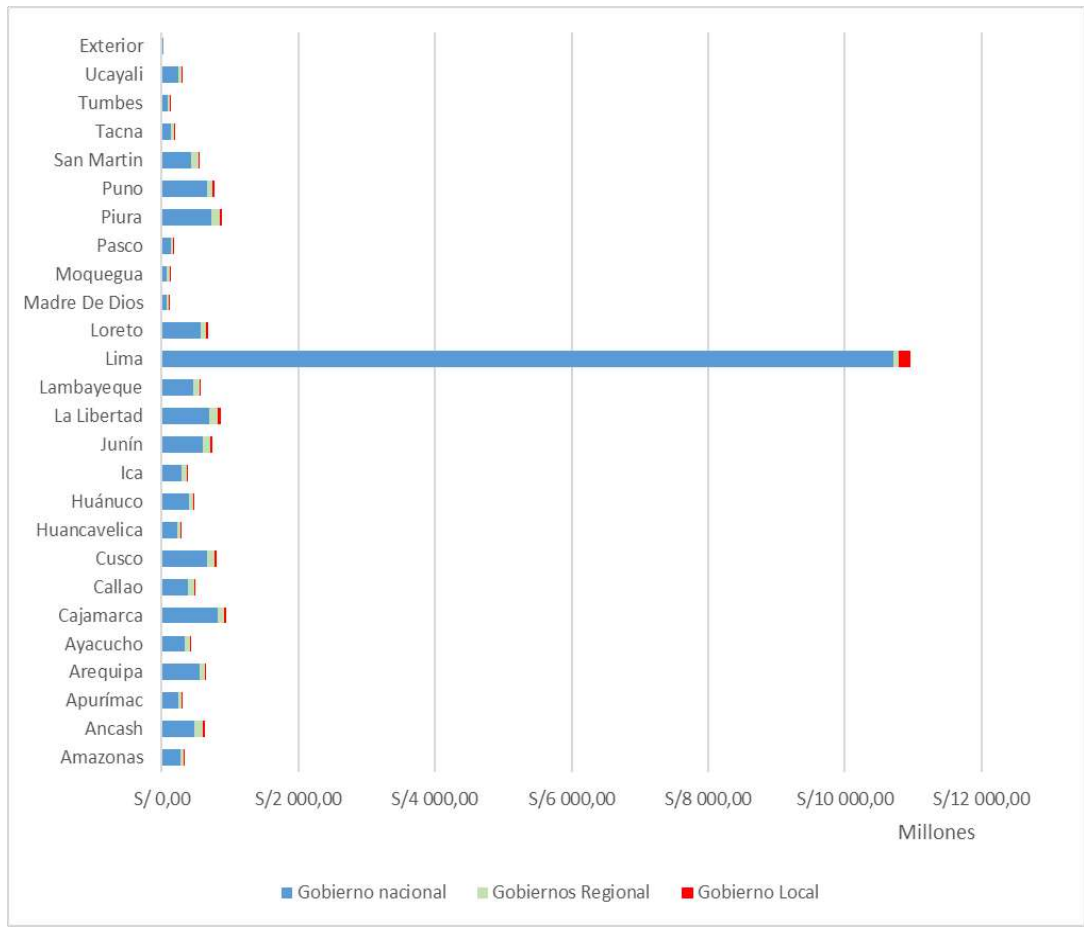

Es preciso analizar los entes gubernamentales que administraron el presupuesto nacional con relación al COVID 19, de los que se desagrega la Tabla 3, donde se destina al Ministerio de Desarrollo e Inclusión Social el 35,2\% del presupuesto, con genérica de gasto orientada a bienes y servicios, donaciones y transferencias, adquisiciones de activos no financieros y otros gastos en subvenciones a personas naturales (bonos), significando este último la mayor 
cantidad. El 34,62\% del presupuesto fue asignado al Ministerio de Trabajo y Promoción del Empleo, utilizando este presupuesto en las genéricas de gasto de bienes y servicios, donaciones y transferencias, adquisiciones de activos no financieros y otros gastos principalmente, donde se consideró las subvenciones financieras, que son gastos de ayuda estatal a personas naturales (bonos). El 16,68\% al Ministerio de Salud, en la genérica de gasto de personal y obligaciones sociales, donaciones y transferencias, adquisición de activos no financieros, otros gastos y principalmente bienes y servicios (para la adquisición de equipos y suministros médicos; y la contraprestación de profesionales de la salud). Asignación importante también recibió el Ministerio de Educación con un 4,13\%, sobresaliendo en esta ejecución la adquisición de equipos para instalaciones educativas. 


\section{Tabla 3.}

Asignación y ejecución presupuestal COVID-19, por entes ejecutores.

\begin{tabular}{|c|c|c|c|}
\hline Sector & Presupuesto asignado & Presupuesto ejecutado & Avance \% \\
\hline Presidencia Consejo Ministros & $\mathrm{S} / 214298609,00$ & $S / 142372365,00$ & $66 \%$ \\
\hline Cultura & $\mathrm{S} / 329324,00$ & $\mathrm{~S} / 275095,00$ & $84 \%$ \\
\hline Poder Judicial & S/14 125437,00 & S/12 057486,00 & $85 \%$ \\
\hline Ambiental & S/18 099 106,00 & S/10 210027,00 & $56 \%$ \\
\hline Justicia & S/45 105687,00 & S/38 474067,00 & $85 \%$ \\
\hline Interior & S/388 199 374,00 & $S / 324545562,00$ & $84 \%$ \\
\hline Relaciones Exteriores & S/41 555 288,00 & $\mathrm{S} / 36456808,00$ & $88 \%$ \\
\hline Economía y Finanzas & S/230 731972,00 & S/165 944724,00 & $72 \%$ \\
\hline Educación & S/840 618 935,00 & $\mathrm{S} / 226487565,00$ & $27 \%$ \\
\hline Salud & $S / 3395674433,00$ & S/2 416523734,00 & $71 \%$ \\
\hline Trabajo y Promoción del Empleo & S/7 050411020,00 & S/6 136220348,00 & $87 \%$ \\
\hline Agricultura & S/19 253116,00 & S/16 083390,00 & $84 \%$ \\
\hline Energía y Minas & $\mathrm{S} / 172293,00$ & $\mathrm{~S} / 172293,00$ & $100 \%$ \\
\hline Contraloría General & $\mathrm{S} / 31024201,00$ & $\mathrm{~S} / 23405013,00$ & $75 \%$ \\
\hline Defensoría del Pueblo & S/3 207748,00 & $S / 1452431,00$ & $45 \%$ \\
\hline Junta Nacional de Justicia & $S / 39765,00$ & S/39 764,00 & $100 \%$ \\
\hline Ministerio Publico & $S / 3650,00$ & $S / 3650,00$ & $100 \%$ \\
\hline Defensa & S/488 804 135,00 & $\mathrm{S} / 328570512,00$ & $67 \%$ \\
\hline Registro Nacional de Identificación y Estado Civil & S/30 467000,00 & S/10 735951,00 & $35 \%$ \\
\hline Comercio Exterior y Turismo & S/61 059099,00 & S/57 442 148,00 & $94 \%$ \\
\hline Transportes y Comunicaciones & S/305 574682,00 & $\mathrm{~S} / 120038524,00$ & $39 \%$ \\
\hline Vivienda Construcción y Saneamiento & $\mathrm{S} / 12516282,00$ & $S / 10960848,00$ & $88 \%$ \\
\hline Producción & $\mathrm{S} / 28890111,00$ & S/24 598167,00 & $85 \%$ \\
\hline Mujer y Poblaciones Vulnerables & S/12 140609,00 & S/5 381220,00 & $44 \%$ \\
\hline Desarrollo e Inclusión Social & S/7 131231 608,00 & S/6 344629169,00 & $89 \%$ \\
\hline Total & $\mathrm{S} / 20363533484,00$ & $S / 16453080861,00$ & $81 \%$ \\
\hline
\end{tabular}

Fuente: Adecuado de (MEF, 2020) al 06 de diciembre del 2020.

Existen ministerios que no recibieron ningún tipo de asignación presupuestal por motivo de COVID 19, ver Figura 3, tal es el caso del Tribunal Constitucional, el Fuero Policial, el Congreso de la República, el Jurado Nacional de Elecciones, la Oficina de Procesos Electorales; con un presupuesto relativamente menor tenemos al sector Cultura, de Energía y Minas, Defensoría del Pueblo, Junta Nacional de Justicia, Ministerio Publico y Contraloría General; este 
último ente rector fiscalizador, cuya misión es "Dirigir, supervisar y ejecutar el control a las entidades públicas para contribuir al uso eficaz, eficiente y transparente de los recursos públicos" (Contraloría, 2020).

\section{Figura 3.}

Asignación y ejecución presupuestal COVID-19, por entes ejecutores.

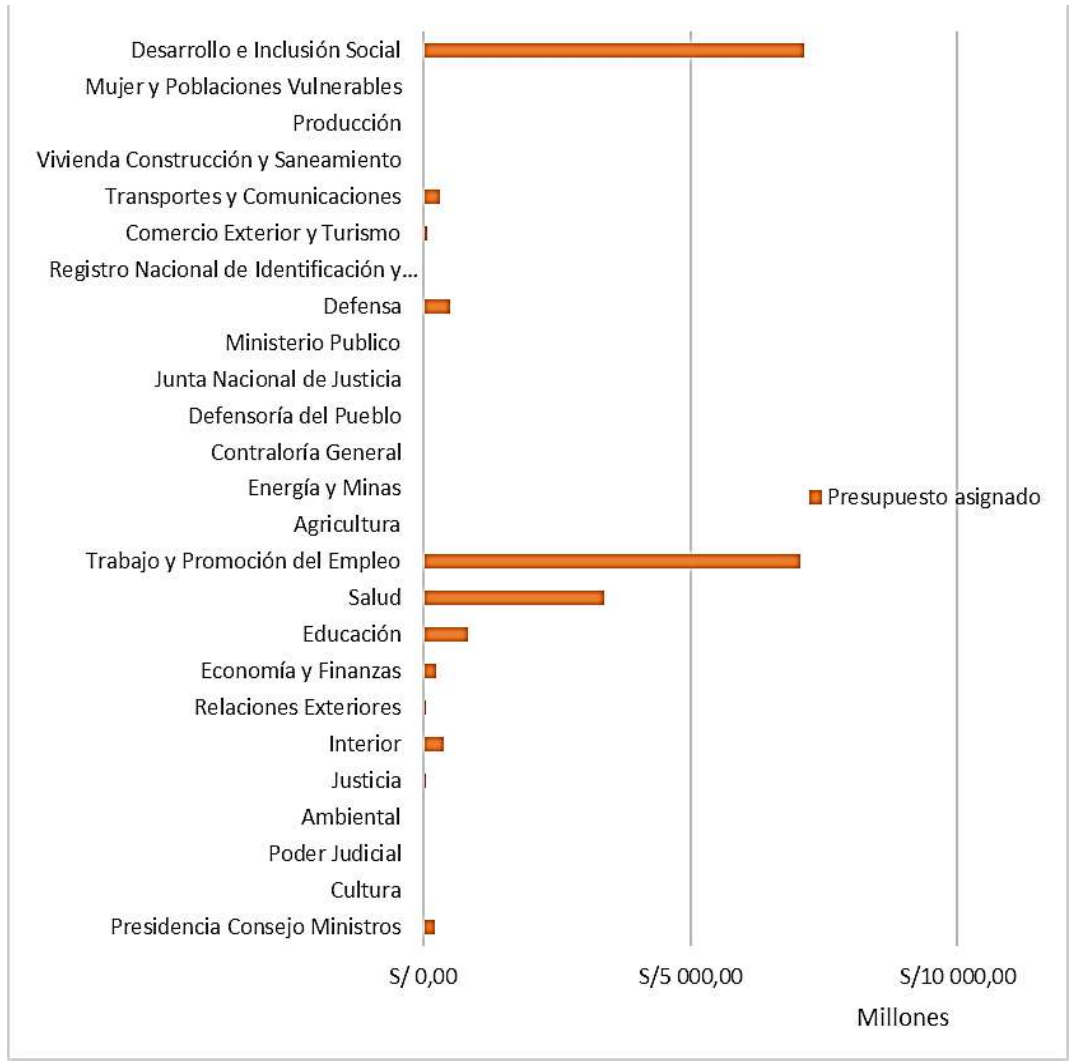

Para que un ente rector tenga cierta eficiencia, debe contar con recursos; el Estado no ha considerado controlar y fiscalizar los gastos abundantes que se realizaron durante la pandemia, los mismos que deben tener una auditoría, por lo que resulta imprescindible tomar acciones que empoderen esta institución. 
Hasta este espacio, ¿de qué nos ha servido tener una herramienta tan valiosa como los ODS de la Agenda 2030? si antes de esta circunstancia, el ciudadano de a pie no ha percibido cambio alguno en las políticas gubernamentales, menos ahora en tiempos de pandemia. Con tanto dinero puesto en arcas de unos pocos, se duda de un trabajo transparente y se espera no acertar, cuando se dice que la mayoría de los movimientos económicos presentan algún índice de corrupción.

\section{PANDEMIA Y CORRUPCIÓN}

Para conocer un poco sobre los niveles de corrupción en los últimos años, se observa la Tabla 4, donde se detalla el perjuicio económico por regiones en los periodos 2017, 2018 y 2019; resultando un total de S/. 32757496705,00 , equivalente a 6551 complejos educativos (inicial, primaria y secundaria) llave en mano, que incluye losas deportivas y laboratorios. Nadie en su sano juicio consideraría justo tanta pérdida económica, existiendo tanta necesidad en los pueblos. 


\section{Tabla 4.}

Perjuicio económico por regiones 2017-2018-2019.

\begin{tabular}{lrrr}
\hline \multicolumn{1}{c}{ Regiones } & \multicolumn{1}{c}{$\mathbf{2 0 1 7 - 2 0 1 8}$} & \multicolumn{1}{c}{$\mathbf{2 0 1 9}$} & \multicolumn{1}{c}{ Total } \\
\hline Amazonas & $\mathrm{S} / 237301435,00$ & $\mathrm{~S} / 311441964,00$ & $\mathrm{~S} / 548743399,00$ \\
Ancash & $\mathrm{S} / 496019635,00$ & $\mathrm{~S} / 804099646,00$ & $\mathrm{~S} / 1300119281,00$ \\
Apurímac & $\mathrm{S} / 251037297,00$ & $\mathrm{~S} / 396436757,00$ & $\mathrm{~S} / 647474054,00$ \\
Arequipa & $\mathrm{S} / 677855322,00$ & $\mathrm{~S} / 966911061,00$ & $\mathrm{~S} / 1644766383,00$ \\
Ayacucho & $\mathrm{S} / 322128358,00$ & $\mathrm{~S} / 493473022,00$ & $\mathrm{~S} / 815601380,00$ \\
Cajamarca & $\mathrm{S} / 412879456,00$ & $\mathrm{~S} / 656557473,00$ & $\mathrm{~S} / 1069436929,00$ \\
Callao & $\mathrm{S} / 706361813,00$ & $\mathrm{~S} / 1132875253,00$ & $\mathrm{~S} / 1839237066,00$ \\
Cusco & $\mathrm{S} / 412374975,00$ & $\mathrm{~S} / 768255095,00$ & $\mathrm{~S} / 1180630070,00$ \\
Huancavelica & $\mathrm{S} / 311206870,00$ & $\mathrm{~S} / 438183493,00$ & $\mathrm{~S} / 749390363,00$ \\
Huánuco & $\mathrm{S} / 227259832,00$ & $\mathrm{~S} / 391628493,00$ & $\mathrm{~S} / 618888325,00$ \\
Ica & $\mathrm{S} / 161529051,00$ & $\mathrm{~S} / 357212754,00$ & $\mathrm{~S} / 518741805,00$ \\
Junín & $\mathrm{S} / 446029704,00$ & $\mathrm{~S} / 712076756,00$ & $\mathrm{~S} / 1158106460,00$ \\
La Libertad & $\mathrm{S} / 309006290,00$ & $\mathrm{~S} / 600175267,00$ & $\mathrm{~S} / 909181557,00$ \\
Lambayeque & $\mathrm{S} / 473881392,00$ & $\mathrm{~S} / 705513000,00$ & $\mathrm{~S} / 1179394392,00$ \\
Lima & $\mathrm{S} / 1565327830,00$ & $\mathrm{~S} / 10359826254,00$ & $\mathrm{~S} / 11925154084,00$ \\
Loreto & $\mathrm{S} / 258214080,00$ & $\mathrm{~S} / 476018452,00$ & $\mathrm{~S} / 734232532,00$ \\
Madre de Dios & $\mathrm{S} / 47394397,00$ & $\mathrm{~S} / 133854599,00$ & $\mathrm{~S} / 181248996,00$ \\
Moquegua & $\mathrm{S} / 182261428,00$ & $\mathrm{~S} / 234427154,00$ & $\mathrm{~S} / 416688582,00$ \\
Pasco & $\mathrm{S} / 211929978,00$ & $\mathrm{~S} / 320850572,00$ & $\mathrm{~S} / 532780550,00$ \\
Piura & $\mathrm{S} / 612053680,00$ & $\mathrm{~S} / 1039931333,00$ & $\mathrm{~S} / 1651985013,00$ \\
Puno & $\mathrm{S} / 350761479,00$ & $\mathrm{~S} / 666297392,00$ & $\mathrm{~S} / 1017058871,00$ \\
San Martin & $\mathrm{S} / 273447721,00$ & $\mathrm{~S} / 463249148,00$ & $\mathrm{~S} / 736696869,00$ \\
Tacna & $\mathrm{S} / 173177828,00$ & $\mathrm{~S} / 277798435,00$ & $\mathrm{~S} / 450976263,00$ \\
Tumbes & $\mathrm{S} / 104488990,00$ & $\mathrm{~S} / 181277674,00$ & $\mathrm{~S} / 285766664,00$ \\
Ucayali & $\mathrm{S} / 236531181,00$ & $\mathrm{~S} / 408665636,00$ & $\mathrm{~S} / 645196817,00$ \\
Total & $\mathrm{S} / \mathbf{9 4 6 0 4 6 0 0 2 2 , 0 0}$ & $\mathrm{S} / \mathbf{2 3} \mathbf{2 9 7} \mathbf{0 3 6} \mathbf{6 8 3 , 0 0}$ & $\mathrm{S} / \mathbf{3 2} \mathbf{7 5 7} \mathbf{4 9 6 7 0 5 , 0 0}$ \\
\hline & & &
\end{tabular}

Fuente: Adaptado de (Shack, Nelson; Pérez, Jenifer; Portugal, 2020).

En medio de la pandemia, una crisis económica que no da tregua y con un gobierno contra las cuerdas por la corrupción, Perú se encamina nuevamente a elecciones y a tener un gobierno legítimo elegido en las urnas que pueda enfrentar su difícil presente y futuro (Molina Monasterios, 2020). Resulta solemne y vital elegir personas con capacidad y moral suficiente para que no ocurran pérdidas económicas, como las del Figura 4, donde ninguna región de nuestro país se salva de la corrupción. Como en todas las líneas, Lima y Callao precede como foco infeccioso del virus de la corrupción con un $42 \%$, 
seguido de Arequipa y Piura con 5\%, de Áncash, Cusco, Junín y Lambayeque con un 4\%; en menor intensidad tenemos a las regiones de Madre De Dios, Tumbes, Moquegua, Tacna con un $1 \%$ promedio.

\section{Figura 4.}

Perjuicio económico por regiones 2017-2018-2019.

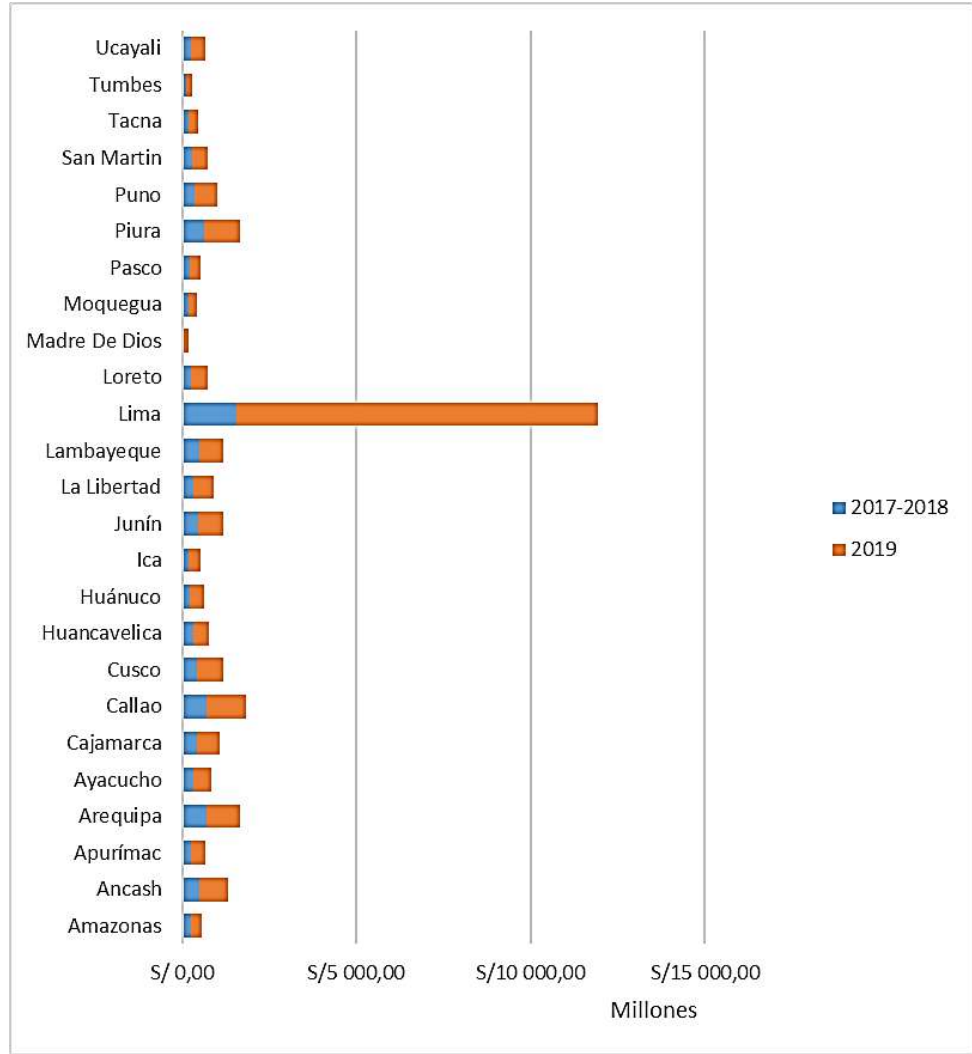

Los sectores que más se han sentido perjudicados con este problema social de la corrupción, de acuerdo al análisis del periodo 2019 , son Transporte y comunicaciones con un $25 \%$, Salud con un 16,2\% y Educación con un 15,6\%, otros 13,9\%, ver Tabla 5. 


\section{Tabla 5.}

Perjuicio económico por principales sectores 2019.

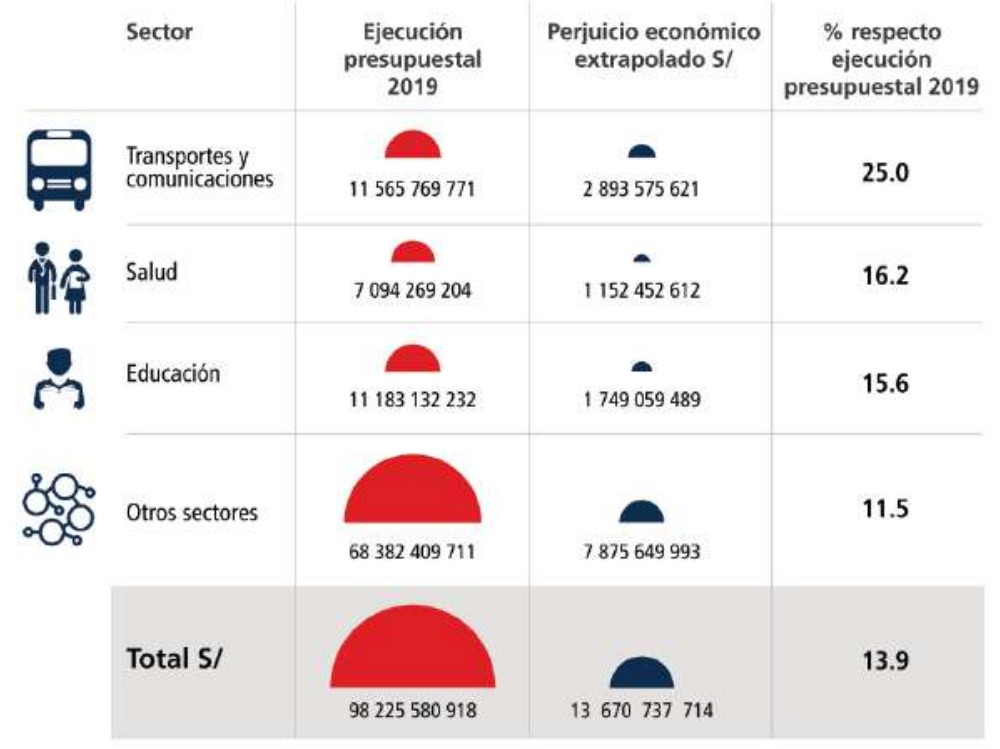

Fuente: (Shack, Nelson; Pérez, Jenifer; Portugal, 2020).

La corrupción dificulta el acceso igualitario a bienes y servicios esenciales, y afecta, principalmente, la vida, la salud y otros importantes derechos de los ciudadanos en situación de pobreza y pobreza extrema, así como de quienes se encuentran en algún especial contexto de vulnerabilidad (Madrid Valerio \& Palomino Ramírez, 2020). Esto ocasiona consecuencias catastróficas en la administración pública, obstruyendo su finalidad y generando descrédito.

El control de los recursos, no sólo está en manos de la sociedad, existe diversos sistemas de fiscalización competentes. Dicha tarea es de la Contraloría General de la República y del Ministerio Público, que tienen una capacidad limitada de respuesta ante la variedad de órganos estatales involucrados en el gasto ocasionado durante la pandemia, (Madrid Valerio \& Palomino Ramírez, 2020), 
presentan debilidad por su actuación posterior, sin capacitad de intervención inmediata. La mejor barrera contra la corrupción en tiempos de coronavirus es estar bien informados (Castro-Martínez, 2020), esta información debe procurar ser periódica, confiable y autónoma, más en situaciones de emergencia (Terziev Venelin; Marin Petrov, 2020).

Las diversas denuncias presentadas durante la pandemia, perturban el pensamiento de buena fe de cualquier ciudadano; leer noticieros con titulares como: "Fiscalía registra más de 1,800 denuncias de presuntos actos de corrupción en pandemia" (Gestión, 2020), estremece; la adquisición ineficiente, hasta la distribución injusta de diversos equipos y víveres, etc.; "Corrupción en tiempos de cuarentena: delitos contra la administración pública en el caso de las canastas familiares" (Pólemos, 2020), un presupuesto relativamente pequeño, frente a todos los gastos realizados en pandemia, que no pudo ser administrado sin reclamos y denuncias, ¿cómo no acusarlos? si lo beneficiados en algunos casos fueron familiares directos de las autoridades.

"La corrupción también mata" (Connectas, 2020), cuando miles de funcionarios tienen bajo su responsabilidad bregar contra la pandemia, mitigar el dolor y velar por el bien común, dejando de lado su deber, buscan enriquecerse a cuestas del sufrimiento del pueblo, claro que la corrupción mata; no hay ejemplo ni en las personas que supuestamente imparten justicia "Corrupción en los tiempos de covid19: Caso Compras de gel por la PNP de Chiclayo" (Chiok Gonzales, 2020); cuando el que lucha contra la delincuencia, comete delito ¿qué hacer?

"Arranca Perú y los riesgos de la corrupción en obras publicas" (Comitre, 2020); así como Invierte Perú, ambas estrategias políticas que sin transparencia han ensombrecido su finalidad pública, "Piden investigar corrupción en compras del gobierno regional por pandemia" (Tiempo, 2020), es una solicitud que debe ser de oficio; los 
entes de control tienen arduo trabajo, al margen de aplicar un control posterior, su responsabilidad es cuidar el bien jurídico del Estado, en este caso nuestros recursos económicos.

Muchas de estas denuncias, han amparado su defensa en el principio de buena fe, esta regulación gubernamental tiene la finalidad de lograr objetivos para el desarrollo en común. El punto de por qué la buena fe es tan importante en un contrato es evitar incumplimientos y reducir pérdidas por ambas partes. Esto puede ser prácticamente un esfuerzo para prevenir la corrupción (Hamzah, 2020). Actuar de buena fe, implica moralidad, la que se logra con educación y cultura; para vencer la corrupción no hay que hacer algo desconocido o extraordinario, todos los males endebles de cualquier nación se vencen con una sola estrategia, educación.

\section{CONCLUSIONES}

La corrupción es el abuso del poder público para obtener un beneficio en particular, un fenómeno social que no permite el desarrollo sostenible de nuestro país; que invade el bien jurídico de la administración pública; su sanción está prescrito en nuestro ordenamiento jurídico y tiene carácter administrativo, civil y penal.

La lucha contra la corrupción, implica un enfoque integral, con participación colectiva de autoridades, funcionarios y ciudadanos; la transparencia de la información es vital y la rendición de cuentas concreta ese principio, adoptar medidas legales y políticas estratégicas para la fiscalización, resultan relevantes en estos tiempos de pandemia, ya que la ejecución de presupuesto ha sido abrumante.

La asignación presupuestal a nivel de Gobierno Nacional, Regional y Local, ascendió a S/.22 864873 138.00, a la fecha del estudio 06 de diciembre de 2020, su ejecución se acercaba al $80 \%$; casi el $90 \%$ del presupuesto fue administrado por el Gobierno Nacional. La distribución por regiones favoreció a Lima en un total del 53\%, seguido de Cajamarca y Piura con un 4\%. El dinero distribuido en el 
Gobierno Nacional fue en mayor parte para el Ministerio de Desarrollo e Inclusión Social con 35,2\%, seguido del Ministerio de Trabajo y Promoción del Empleo con 34, 62\%, ambas instituciones administraron las subvenciones económicas (bonos) a personas naturales. También recibieron aporte significativo los Ministerios de Salud y Educación con un 16,68\% y 4,13\% respectivamente.

El perjuicio económico del 2017 al 2019, según la Contraloría General de la Republica ascendió a S/. 32757496 705,00; siendo el mayor importe de pérdida para Lima y Callao, seguido de Arequipa y Piura, en los sectores de transporte y comunicaciones, salud y educación.

Diversas denuncias surgieron en tiempos de pandemia, el control posterior queda pendiente por parte de los entes recortes; actuar de buena fe y con transparencia es una premisa, que se asocia a la moralidad; la educación es una estrategia sólida para vencer la corrupción.

Hace falta más que una herramienta de gestión como los Objetivos de Desarrollo Sostenible de la Agenda 2030, que no genera impacto respecto a esta problemática, por la forma sutil de su estrategia para eliminarla. Se requiere un tratamiento transversal ya que su presencia perturba la mayoría, por no decir todos los ODS, sin esa premisa, no se logrará lo ansiado que es la sostenibilidad de las naciones.

\section{REFERENCIAS BIBLIOGRÁFICAS}

Begovic, B. (2005). Corrupción: conceptos, tipos, causas y consecuencias. Centro Para La Apertura y El Desarrollo de America Latina, 3(26), 8.

https://www.cadal.org/Documentos/documento_26.pdf\%0Ah ttp://www.offnews.info/downloads/corrupcion_conceptos_ca dal.pdf

Carhuavilca Bonett, Dante; Sánchez Aguilar, Aníbal; Robles Luis, 
José; Meza Meza, H. (2020). Producto bruto interno trimestral. Informe Técnico INEI, 4.

https://www.inei.gob.pe/media/MenuRecursivo/boletines/inf orme-tecnico-pbi-iii-trim-2020.pdf

Castro-Martínez, J. A. (2020). Corrupción de la desinformación en tiempos de coronavirus. Revista de Información Científica Para La Dirección En Salud. INFODIR, 32, 1-9. http://www.revinfodir.sld.cu/index.php/infodir/article/view/8 02

Chiok Gonzales, J. (2020, May 31). Corrupción en los tiempos de covid-19: Caso Compras de gel por la PNP de Chiclayo | Javier Miguel Chiok - IUS 360. IUS 360, 1-4. https://ius360.com/actualidad/corrupcion-en-los-tiempos-decovid-19-caso-compras-de-gel-por-la-pnp-de-chiclayojavier-miguel-chiok/

Comitre, P. (2020, July 8). Arranca Perú y los riesgos de la corrupción en obras publicas. Conexión Esan, 1-4. https://www.esan.edu.pe/conexion/actualidad/2020/07/08/arr anca-peru-y-los-riesgos-de-la-corrupcion-en-obras-publicas/

Connectas. (2020, December 8). La corrupcióntambién mata.

Connectas, 1-12. https://www.connectas.org/pandemia-lacorrupcion-tambien-mata/

Contraloría. (2020). Portal de Transparecia contraloría General de la República (p. 1).

https://www.contraloria.gob.pe/wps/wcm/connect/CGRNew/ as_contraloria/as_portal/Conoce_la_contraloria/conoceContr aloria/mision/

Gestión. (2020, November 9). Fiscalía registra más de 1,800 denuncias de presuntos actos de corrupción en pandemia Hasta. 1-5. https://gestion.pe/peru/fiscalia-registra-mas-de1800-denuncias-de-presuntos-actos-de-corrupcion-enpandemia-nndc-noticia/

Gobierno de Chile. (2017). Informe de diagnóstico e implementación de la agenda 2030 y los objetivos de desarrollo sostenible en 
Chile. Consejo Nacional Para La Implementación de La Agenda 2030 Para El Desarrollo Sostenible (ODS)

Ministerio de Desarrollo Social, 386.

http://www.chileagenda2030.gob.cl/storage/docs/Diagnostic o-Inicial_2.0_Agenda2030-ODS_2017.pdf

Hamzah. (2020). Civil law agreement and its implication on regulation for prevention of corruption within COVID-19 pandemic. Journal of Social Studies Education Research, 11(3), 156-176.

Khasiani, K., Koshima, Y., \& Mfombouot, A. (2020). Serie especial sobre la COVID-19 - Controles de la ejecución presupuestaria para mitigar el riesgo de corrupción en el gasto por la pandemia. Fiscal Affairs, 1-11.

Madrid Valerio, C., \& Palomino Ramírez, W. (2020). Oportunidades de corrupción y pandemia: El compliance gubernamental como un protector eficaz al interior de las organizaciones públicas. Desde El Sur, 12(1), 213-239. https://doi.org/10.21142//DES-1201-2020-0014

MEF. (2020). Transaparencia Económica Perú (p. 2020). https://apps5.mineco.gob.pe/coronavirus/Navegador/default.a $\operatorname{spx}$

Molina Monasterios, S. (2020). Bolivia en medio de la pandemia, la corrupción y los Avengers. Question/Cuestión, 1(junio), e389. https://doi.org/10.24215/16696581e389

Naciones Unidas/CEPAL. (2019). La Agenda 2030 y los Objetivos de Desarrollo Sostenible: una oportunidad para América Latina y el Caribe. Objetivos, metas e indicadores mundiales. In Publicación de las Naciones Unidas. https://repositorio.cepal.org/bitstream/handle/11362/40155/2 4/S1801141_es.pdf

Pólemos. (2020, June 21). Corrupción en tiempos de cuarentena: delitos contra la administración pública en el caso de las canastas familiares. Pólemos, Portal Jurídico Interdisciplinario, 1-14. https://polemos.pe/corrupcion-en- 
tiempos-de-cuarentena-delitos-contra-la-administracionpublica-en-el-caso-de-las-canastas-familiares/

Ramón R, J. G. (2014). Corrupción, Ética Y Función Pública En El Perú. Quipukamayoc, 22(41), 59-73. https://d1wqtxts1xzle7.cloudfront.net/52785688/1006935133-1-PB.pdf?1493014351=\&response-contentdisposition=inline \%3B+filename\%3DROLE_OF_CORRuPT ION_ETHICS_AND_PuBLIC_FuN.pdf $\&$ Expires $=16034811$ $01 \&$ Signature $=$ Vok5GWSnKXlaQYrz9SMY2QI30vwGMYZ9rc9YuuIK8xpapgq 49

Rodríguez Collao, L. (2004). Delimitación del concepto penal de corrupción. Revista de Derecho, 5(7), 339-359. https://doi.org/1254251526

Rodriguez Soria, Beatriz; Jacotte Simancas, Coro; Moreno Fernández, José Ramón; Álvarez Domínguez, J. R. (2016). ¿QUÉ DESARROLLO QUEREMOS? LA AGENDA POST 2015 Y LOS OBJETIVOS DE DESARROLLO SOSTENIBLE (U. de Z. Cátedra de Cooperación para el Desarrollo (ed.); Red Españo). https://d1wqtxts1xzle7.cloudfront.net/53527401/ACTAS_C ONGRESO_RIED_WEB.pdf?1497548884=\&responsecontentdisposition=inline\%3B+filename\%3DMigracion_y_Desarrol lo_mas_integracion_e.pdf\&Expires=1596091715\&Signature =gpDPVBMnntxWrJfQR7d6 O9L6bL4VWLLKYRuvJovG hOm

Shack, Nelson; Pérez, Jenifer; Portugal, L. (2020). Cálculo del tamaño de la corrupción y la inconducta funcional en el Perú: Una aproximación exploratoria. Documento Política En Control Gubernamental. Contraloría General de La República. Lima, Perú. https://doc.contraloria.gob.pe/estudiosespeciales/documento_trabajo/2020/Calculo_de_la_Corrupci on_en_el_Peru.pdf

Soto Posada, G. (2018). La corrupción, una pandemia en Colombia. 
Comunicación, 39, 53-63. https://doi.org/10.18566/comunica.n39.a04

Tapia Gutiérrez, A. (2016). Empresas y Derechos Humanos. Derecho y Realidad, 2(24).

https://doi.org/10.19053/16923936.v2.n24.2014.4527

Terziev Venelin; Marin Petrov, G. (2020). Increasing the risk of corruption activities during a COVID-19 pandemic.

Osteoarthritis and Cartilage, 28(2), 1-43. http://journals.sagepub.com/doi/10.1177/1120700020921110 \%0Ahttps://doi.org/10.1016/j.reuma.2018.06.001\%0Ahttps:// doi.org/10.1016/j.arth.2018.03.044\%0Ahttps://reader.elsevie r.com/reader/sd/pii/S1063458420300078?token=C039B8B13 922A2079230DC9AF11A333E295FCD8

Tiempo. (2020, August 26). Piden investigar corrupción en compras del gobierno regional por pandemia. Diario El Tiempo de Piura, 1-8. https://www.rcrperu.com/piden-investigarcorrupcion-en-compras-del-gobierno-regional-por-pandemia/

UNODC. (2018). Corrupción y desarrollo sostenible. Oficina de Naciones Unidas Contra Las Drogas y El Delito, 1. http://www.anticorruptionday.org/documents/actagainstcorru ption/print/corr18_fs_DEVELOPMENT_es.pdf 